\title{
CDISC SDTM Mechanical Circulatory Support Placement Timing Terminology
}

National Cancer Institute

\section{Source}

National Cancer Institute. CDISC SDTM Mechanical Circulatory Support Placement

Timing Terminology. NCI Thesaurus. Code C101845.

Terminology associated with the mechanical circulatory support placement timing codelist of the Clinical Data Interchange Standards Consortium (CDISC) Study Data Tabulation Model (SDT M). 